University of Nebraska - Lincoln

DigitalCommons@University of Nebraska - Lincoln

Faculty Publications in Computer \& Electronics Electrical \& Computer Engineering, Department Engineering (to 2015)

2011

\title{
Mobile WiMAX Throughput and Delay Measurements in Railroad Environment
}

\author{
Puttipong Mahasukhon \\ University of Nebraska-Lincoln, pmahasukhon@huskers.unl.edu \\ Hamid Sharif \\ University of Nebraska-Lincoln, hsharif@unl.edu \\ Michael Hempel \\ University of Nebraska Lincoln, mhempel2@unl.edu \\ Tao Ma \\ University of Nebraska Lincoln, tma@unlnotes.unl.edu \\ Pradhumna Shrestha \\ University of Nebraska Lincoln, plshrestha@unInotes.unl.edu
}

Follow this and additional works at: https://digitalcommons.unl.edu/computerelectronicfacpub

Part of the Computer Engineering Commons

\begin{abstract}
Mahasukhon, Puttipong; Sharif, Hamid; Hempel, Michael; Ma, Tao; and Shrestha, Pradhumna, "Mobile WiMAX Throughput and Delay Measurements in Railroad Environment" (2011). Faculty Publications in Computer \& Electronics Engineering (to 2015). 101.

https://digitalcommons.unl.edu/computerelectronicfacpub/101

This Article is brought to you for free and open access by the Electrical \& Computer Engineering, Department of at DigitalCommons@University of Nebraska - Lincoln. It has been accepted for inclusion in Faculty Publications in Computer \& Electronics Engineering (to 2015) by an authorized administrator of DigitalCommons@University of Nebraska - Lincoln.
\end{abstract}




\title{
Mobile WiMAX Throughput and Delay Measurements in Railroad Environment
}

\author{
Puttipong Mahasukhon, Hamid Sharif, Michael Hempel, Tao Ma, and Pradhumna Lal Shrestha \\ Department of Computer and Electronics Engineering \\ University of Nebraska-Lincoln \\ Omaha, NE, USA \\ \{pmahasukhon, hsharif, mhempel, tma, plshrestha\}@unlnotes.unl.edu
}

\begin{abstract}
The railroad industry in North America is heavily involved in improving the broadband wireless connectivity of their railroad operations. We propose our approach of establishing a Mobile WiMAX test bed as a part of our collaborations with the Federal Railroad Administration (FRA) for investigating the performance of current and upcoming broadband wireless technologies in a railroad environment. The focus is on studying the impact of mobility on the wireless system throughput for moving trains at high velocities. Our goal is to provide a comprehensive measurement that will assist others in understanding Mobile WiMAX performance and guiding future network deployment. In this paper, we describe details of our test bed and discuss test results obtained from our laboratory and test bed. Based on our measurements, we can achieve a throughput of approximately 2.8 Mbps downlink and 2 Mbps uplink with the most robust modulation scheme, a maximum distance of 7.5 kilometers, and a speed of $60 \mathrm{mph}$, making Mobile WiMAX a promising candidate for large-scale deployments in environments such as the railroad industry.
\end{abstract}

Keywords-Mobile WiMAX performance; IEEE 802.16e2005; test bed; measurement; wireless; mobility; wireless in railroads

\section{INTRODUCTION}

Worldwide Interoperability for Microwave Access (WiMAX) is one of the most promising network technologies for providing wireless broadband access to mobile users. Fixed WiMAX is based on the IEEE 802.162004 standard [1], while Mobile WiMAX is based on the IEEE 802.16e-2005 standard [2] which aims to provide highspeed, high-bandwidth, and high-capacity Broadband Wireless Access (BWA) over long distances for residential and mobile users as well as enterprise applications. The physical layer of WiMAX was designed with much influence from Wi-Fi, especially IEEE-802.11a. Although many aspects of the two technologies are different due to the inherent differences in their purpose and application, some of their basic building blocks are very similar. Recently, IEEE 802.16-2009 was released as a revision and consolidation of IEEE 802.16-2004, IEEE 802.16e-2005, and IEEE 802.16f2005.

Our research team at the University of Nebraska's Advanced Telecommunication Engineering Lab (TEL) began a study of different standards-based wireless network technologies and their feasibility to use in a railroad environment for the Federal Railroad Administration (FRA) [3] and in close collaboration with all major railroad companies in the U.S. and Canada. Any desired technology would have to support mobility, provide large coverage areas, high throughput, low latency, and be robust in the presence of noise and interference. In the first phase, we focused on wireless local area network (WLAN) technology and found that it works very well even in high velocity mobile scenarios. A detailed report on our 3.5-mile Wi-Fi test bed is published in [4]. Even though we achieved a maximum mobile communication distance of about 1.6 kilometers around an omni-directional antenna, for feasibility in railroad applications, we needed to find a technology that provides larger distances than Wi-Fi. This brought us to our second phase in which we are looking at WiMAX technology because the promise of theoretical WiMAX range for more than 10 miles could dramatically reduce deployment costs of wireless networks.

Due to the lack of availability and the price of Mobile WiMAX equipment, almost all researchers have extensively studied the performance of Mobile WiMAX systems based on either computer simulations or numerical analysis. However, simulations have inherent limitations because they are developed from numerical models and realistic assumptions. There have been few researches conducting actual WiMAX experiments. In [5], the authors did experiments on WiBro (Wireless Broadband), which is a Korean version of a Mobile WiMAX/IEEE 802.16e system. In [6], they analyzed packet error rate (PER) and shown that the Mobile WiMAX was able to achieve a street-level range of 300-2100 meters with the speeds of up to $35 \mathrm{~km} / \mathrm{h}$. In [7], the authors evaluated the video application performance of a Fixed WiMAX system at a few locations. In [8], they presented a measurement of Mobile WiMAX results in a test bed by acquiring a path loss data in a sea port area. By utilizing the measurement data, their throughput analysis was carried out by means of a computer simulator. However, none of them did an experiment on the distance test with high velocity of $65 \mathrm{mph}$ or $100 \mathrm{~km} / \mathrm{h}$.

The rest of this paper is structured as follows. Section 2 provides a quick overview of the IEEE 802.16e-2005 protocol as it relates to Mobile WiMAX air interface. Section 3 describes our equipment and test beds. We present our findings from our laboratory and test beds in section 4 and 5 , respectively. In section 6 , we summarize our findings and provide an overview of our future work. 


\section{OVERVIEW OF MOBILE WIMAX}

This section provides a brief description of the general MAC layer information flow and a more detailed description of the Mobile WiMAX PHY layer features.

\section{A. The Physical Layer (PHY)}

The IEEE 802.16 Working Group originally developed the broadband point-to-multi-point (PMP) standard as a wireless extension from a wired network infrastructure. The IEEE-802.16 standards define four PHY layers, any of which can be used with the media access control (MAC) layer to develop a broadband wireless system. The first approved air interface was based on single carrier transmission with a time division multiple access (TDMA) protocol. The antenna was originally configured for fixed terminal with line-ofsight (LOS) propagation in the 10 to $66 \mathrm{GHz}$ frequency bands. This gives IEEE 802.16 the ability to provide very high capacity links on both uplink (UL) and downlink (DL). The standard was later extended to include an OFDM technique which is robust for non-line-of-sight (NLOS) propagation across the 2 to $11 \mathrm{GHz}$ range. Both time division duplex (TDD) and frequency division duplex (FDD) are supported in the standard. The standard also includes an OFDMA PHY layer with sub-channelization that allows the time and frequency resources to be dynamically allocated among multiple users across the downlink and uplink subframes. Unlike the $3 \mathrm{G}$ systems which have fixed channel bandwidth, Mobile WIMAX defines a Scalable OFDMAbased PHY layer (SOFDMA) where the Fast Fourier Transform (FFT) size is variable and can take any one of the following values: 128, 512, 1024 and 2048. The variable FFT size allows for optimal operation and implementation of the system over a wide range of channel bandwidths of 1.25, 5,10 , and $20 \mathrm{MHz}$ to flexibly address the need for various spectrum allocation and application requirements. When the operating bandwidth increases, the FFT size is also increased to maintain a fixed subcarrier frequency spacing of 10.94 $\mathrm{kHz}$. This ensures that the OFDMA symbol duration is fixed, so that the impact of bandwidth scaling can be minimized for the upper layers.

The first process in the Mobile WiMAX PHY layer is related to forward error correction (FEC), and includes data randomization, channel encoding, rate matching, interleaving, and modulation. Data randomization is performed on all data transmitted on the downlink and uplink, except the Frame Control Header $(\mathrm{FCH})$, using the output of a maximum length shift register sequence initialized at the beginning of every FEC block. The coding method used in the standards as the mandatory scheme is the convolutional coding (CC) and the optional modes like block turbo coding (BTC) or convolutional turbo codes (CTCs) are also supported. All encoded data bits are interleaved by a block interleaver with a block size corresponding to the number of coded bits per the encoded block size (Ncbps). The interleaver is defined by a two-step permutation. The first step ensures that adjacent coded bits are mapped onto nonadjacent sub-carriers. The second step ensures that adjacent coded bits are mapped alternately onto less or more significant bits of the constellation, avoiding long runs of less reliable bits. Then, the bit stream enters the constellation mapper. The constellation is normalized by multiplying the constellation point with the corresponding factor in order to achieve similar average power. The allowed modulation schemes in the downlink and uplink are binary phase shift keying (BPSK), Quaternary PSK (QPSK), 16-quadrature amplitude modulation (QAM), and 64-QAM.

The next process is related to the construction of the OFDM symbol in the frequency domain. In this process, data is mapped into the appropriate sub-channels and sub-carriers. Pilot symbols are inserted into the pilot sub-carriers, which allow the receiver to estimate and track the channel state information (CSI). This stage is also responsible for any space/time encoding for transmission diversity such as MIMO. The final process is related to the conversion of OFDM symbols from the frequency domain to the time domain and eventually to an analog signal that can be transmitted over the air.

\section{B. The Medium Access Control Layer (MAC)}

The MAC was designed to accommodate different PHYs for the different environments with a variety of quality of service (QoS) requirements. The signaling and bandwidth allocation algorithms were designed to support hundreds of users. The services required by the end users can be varied according to their bandwidth and latency requirements, which require the MAC layer protocol to be flexible and effective.

The IEEE 802.16 MAC layer is divided into two sublayers: convergence-specific and common part. First, the convergence-specific sub-layer specifies how to map the transport-layer-specific traffic to the MAC layer. It is flexible enough to efficiently carry any traffic type. The common part sub-layer is independent of the transport mechanism, and responsible for fragmentation and segmentation of MAC service data units (SDUs) into MAC protocol data units (PDUs), QoS control, and scheduling and retransmission of MAC PDUs.

\section{EQUIPMENTS AND TEST ENVIRONMENTS}

\section{A. Mobile WiMAX Equipments}

After IEEE 802.16e-2005 was approved, we put our efforts into the procurement of WiMAX equipment for Mobile WiMAX laboratory and field tests. Unfortunately, this took much longer than expected, because the WiMAX Forum [9] was slow to agree upon certification test procedures. We surveyed many vendors who had previously announced that they were working on Mobile WiMAX equipment. We were interested in two device categories: the Mobile WiMAX base stations and the ASN Gateway. This device search was quite complicated because we could not consider these categories in isolation. Support for the WiMAX Forum Network Working Group's ASN profiles and equipment compatibility was the issue. We had to carefully select from among the available devices and vendors. For our equipment search, profile $B$ and $C$ compliant devices were preferred. Finally, we found two companies, WiNetworks and WiChorus, which provided 


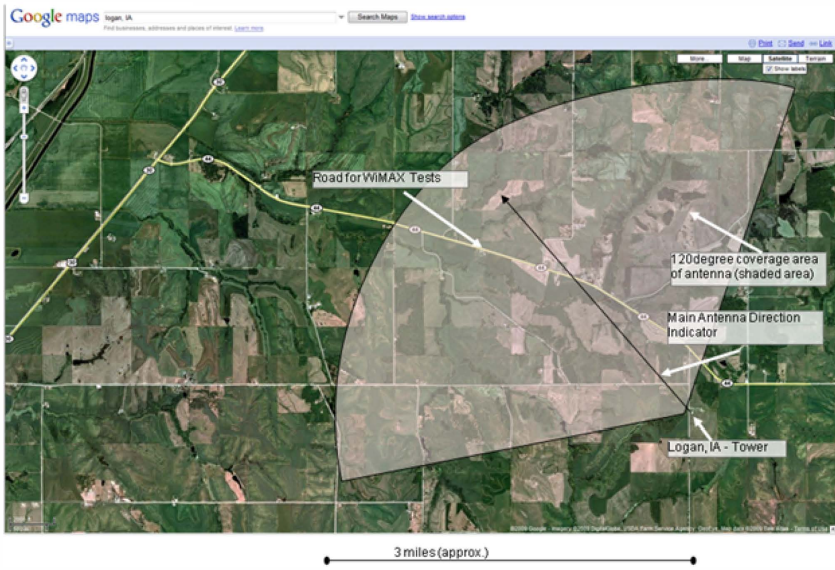

Figure 2. Mobile WiMAX field test location in Logan, Iowa.

solutions that are a perfect match for our requirements. WiNetworks was later acquired by RuggedCom, a Canadian company. We received two RuggedCom WiN-7200 Pico Base Stations, two RuggedCom WiN-7000 Compact Base Stations, and few subscriber stations, and residential gateways. We also purchased a WiChorus SC-20 ASNGateway, which is a small, yet capable ASN Gateway solution.

\section{B. Laboratory}

The ACE-400WB, a Wireless Channel Emulator from Azimuth Systems, was utilized to emulate virtually any wireless channel environment. Instead of transmitting wirelessly over the air, we transmit through the channel emulator. Over the air testing is highly susceptible to a very large number of impairments that are outside of our control. For our laboratory tests, we can have an environment with the channel emulator providing highly reliable testing because all effects that are applied to the RF signal, from path loss to channel coefficients, multipath delays, noise, etc., are fully controllable by the channel emulator. Furthermore, unlike real-world field measurements, experiments in this environment are now repeatable, providing an ideal method for troubleshooting aspects of performance measurements that were not satisfactory or that may show unexpected behavior.

\section{Test Bed in Logan, IA}

To corroborate our initial findings, we developed a dedicated field test environment. To maximize the test effectiveness, we decided to utilize typical microwave tower sites that the railroads operate across the country. After proposing this approach to both Union Pacific and BNSF, both railroads were eager to assist and support us in these tests. Union Pacific is providing access to their tower in Logan, Iowa. This tower is approximately $200 \mathrm{ft}$. tall, and is also located on a hill overlooking the surrounding areas. This provides a height above the test road of approximately 300 feet which we are utilizing for our tests. Our desired antenna height is 250 feet above the test area. The tower therefore exceeds this requirement. Overlaid is an indicator for the

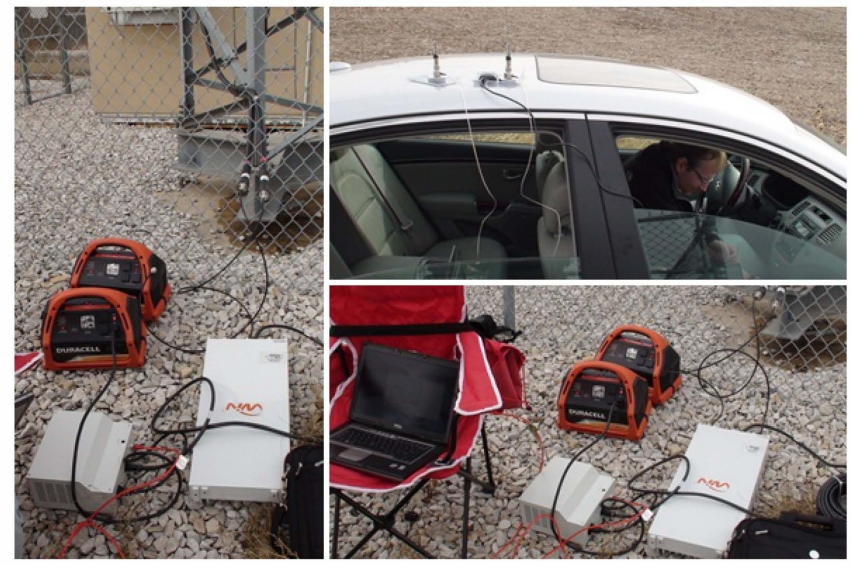

Figure 1. Images from the test bed in Logan, Iowa.

primary antenna orientation and its associated 120 degree antenna coverage sector. The original minimum desired coverage distance of 3 miles is indicated by the radius of the sector arc. The selected road for our tests is Highway 44, also shown in the satellite map. It passes by the tower location and intersects in a westbound direction with Highway 30. Our entire test area stretches from this intersection back to the tower location. Along the entire test road, we can test at velocities of approximately $60 \mathrm{mph}$. Fig. 2 shows our test equipment on a typical test day in Logan, IA.

\section{LABORATORY EXPERIMENTS}

One of our test approaches for Mobile WiMAX performance is laboratory testing. Even before we take the equipment out into the field, we have to determine several important parameters. The most important parameter is the maximum supported communication distance under different channel conditions and device configurations. This is crucial for planning our field tests. Therefore, we need to have a clear understanding of the maximum distances for certain performance requirements. Once we obtained the Mobile WiMAX equipment, we first performed tests in our laboratory using wireless channel emulation and isolating the equipment by using RF enclosures. For the laboratory testing, the idea is not to leak any RF energy into the environment, and not to expose the test equipment to any RF energy from the environment. These tests provided us a better understanding of the approximate distances that Mobile WiMAX can cover under ideal (free-space, interference-free) conditions, as well as emulated real-world conditions. Laboratory test results obtained from the channel emulator and Mobile WiMAX equipment with parameters configured are shown in Table I.

Several modulation schemes have been specified in Mobile WiMAX. To optimize performance, Adaptive Modulation and Coding (AMC) scheme is utilized by the base station to automatically adapt to varying link qualities between the base station and a client device. The base station periodically evaluates these link qualities and chooses the best suitable modulation and coding scheme. We extensively 


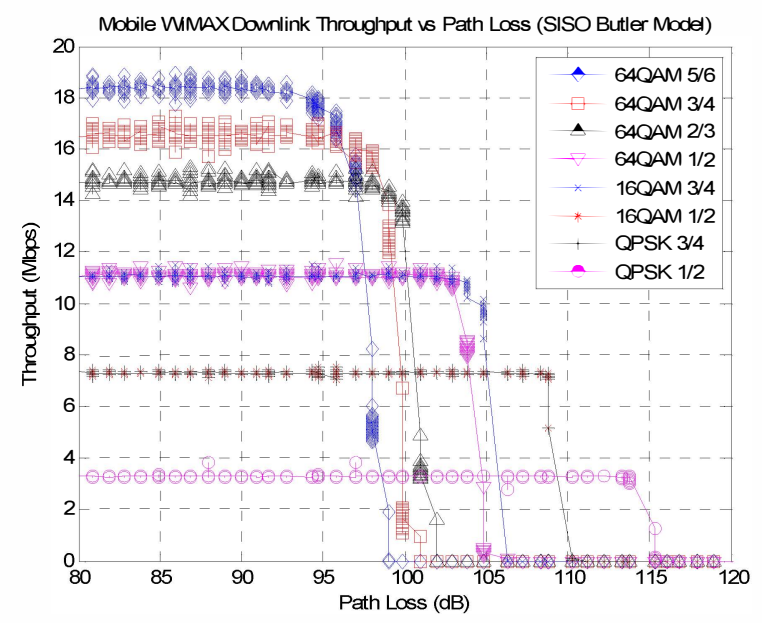

Figure 4. Mobile WiMAX throughput vs. Pass Loss (Downlink) under SISO Butler channel.

tested all the modulation schemes supported by Mobile WiMAX in order to see the performance and range that could be acquired from each modulation. We connected the base station and subscriber station through the wireless channel emulator. The channel model that we chose is the Butler model, which is simple model, allowing us to get the best performance out of our Mobile WiMAX equipment. We finished this effort under all channel conditions in the Butler model by adjusting the attenuator inside the channel emulator as if the distance between the base station and the subscriber station is changed. The base station generated UDP traffic of about $20 \mathrm{Mbps}$, while the subscriber station generated $10 \mathrm{Mbps}$ UPD traffic. We then collected all throughput measurements for each Received Signal Strength Indicator (RSSI) at both devices.

TABLE I. MOBILE WIMAX PARAMETERS USED IN EXPERIMENTS

\begin{tabular}{|l|l|}
\hline \multicolumn{1}{|c|}{ Parameters } & \multicolumn{1}{c|}{ Values } \\
\hline Packet payload & 1,000 bytes \\
Carrier frequency & $2.5 \mathrm{GHz}$ \\
FFT size & 1024 \\
Channel bandwidth & $10 \mathrm{MHz}$ \\
Frame duration & $5 \mathrm{milliseconds}$ \\
Cyclic prefix & $1 / 8$ \\
Sub-carrier frequency spacing & $10.94 \mathrm{kHz}$ \\
Number of OFDMA symbols per frame & $48 \mathrm{symbols}$ \\
DL/UL ratio & $31: 15$ \\
Permutation scheme & PUSC \\
HARQ & Off \\
\hline
\end{tabular}

Fig. 3 and Fig. 4 illustrate the Mobile WiMAX downlink throughput and uplink throughput when the base station and the subscriber station each use a single antenna. This system is termed Single Input Single Output (SISO). As can be expected, the 64-QAM provides high throughput on both downlink and uplink directions, but requires a good receive signal to be able to demodulate in the receiver. In contrast, the QPSK is more robust, but it lowers the throughput. In a working system, the AMC scheme is applied to optimize the throughput performance based on the link quality. If the link

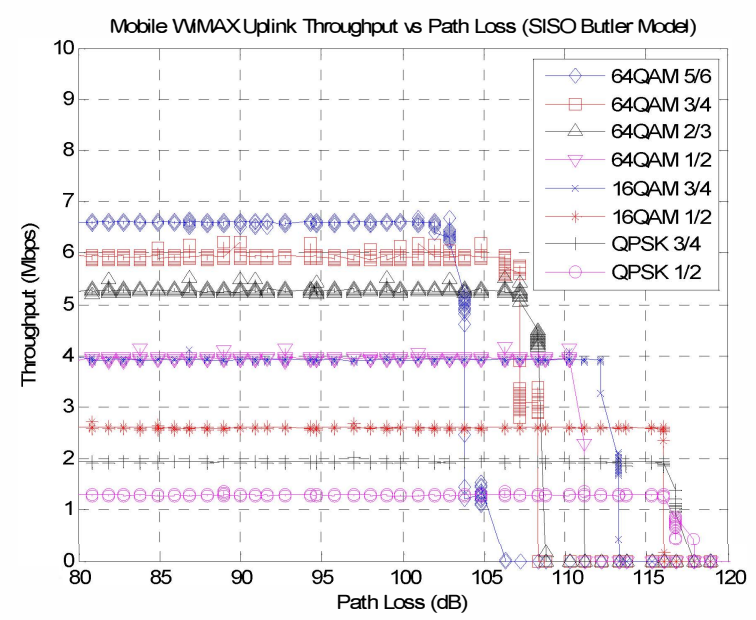

Figure 3. Mobile WiMAX throughput vs. Path Loss (uplink) under SISO Butler channel.

is of sufficient quality, the AMC scheme may be changed towards more throughput potential. If the link is of lower quality than expected, the AMC scheme is adjusted towards a more robust but lower throughput scheme. The uplink throughput is lower than the downlink throughput due to the difference in channel allocation method and the DL/UL ratio. In our laboratory test, we used 31:15 as the DL/UL ratio, which is the default value that obtains from the vendor. Note that in this test the transmit power of both devices was set to $25 \mathrm{dBm}$ and $10 \mathrm{~dB}$ attenuators were inserted to both ends to prevent damage on our wireless channel emulator. The path loss values in Fig. 3 and Fig. 4 are included two $10 \mathrm{~dB}$ attenuators.

With these data, we then selected our test sites, given the constraints of the EBS licenses available to the University of Nebraska. Based on these locations, we then designed test scenarios to be performed once the equipment was installed in Logan, Iowa.

\section{FIELD TEST EXPERIMENTS}

After completing our initial set of laboratory performance measurements, we wanted to experience the performance and general capabilities of Mobile WiMAX in a real-world situation. The distance and coverage testing is one of the most significant tests, since this test is of very high importance to the railroads. We increased the mobile's client distance from the base station and measured the link quality and throughput performance for all locations. In the field test, the QPSK 1/2 modulation scheme is chosen to maximize the Mobile WiMAX coverage. Note that for the field test, the DL/UL ratio was changed from 31:15 to 25:21 to make more balance between the downlink and the uplink throughput, while the other parameters remained the same.

We performed our first field test at the test site in Logan, Iowa, and tried to measure Mobile WiMAX radio distance in a typical rural area. This particular location was chosen because of its representative nature of rural complications in deploying outdoor wireless networks. In most areas, it is open and unobstructed, with only a few segments surrounded 


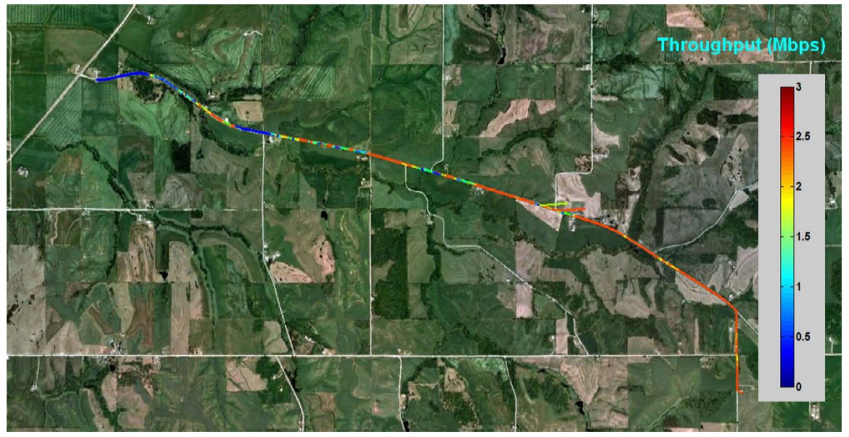

Figure 8. GPS information with downlink throughput results in Logan, IA.
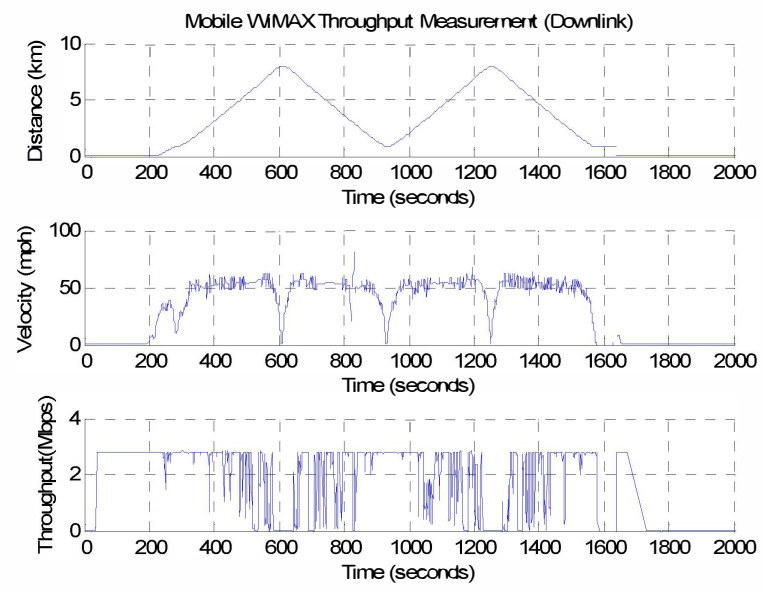

Figure 7. Downlink time domain measurement results in Logan, IA.

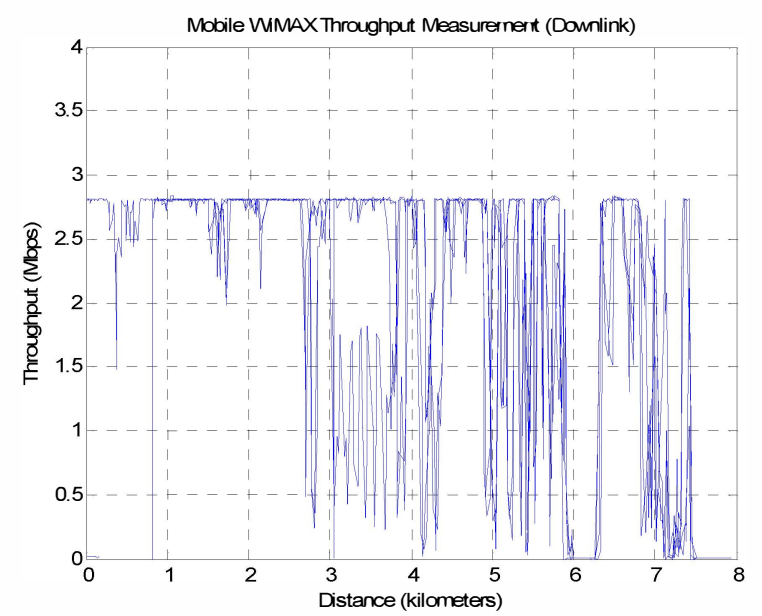

Figure 6. Mobile WiMAX downlink throughput and distance in Logan, IA.

by dense foliage. There is no high building in the vicinity of the test road in this area.

The first test in Logan focused on the mobile downlink performance measurement. The $5 \mathrm{Mbps}$ UDP packet traffic was transmitted from the base station to the mobile client, which was deposited in our test car. The base station's transmit power was set to $30 \mathrm{dBm}$. We mapped GPS

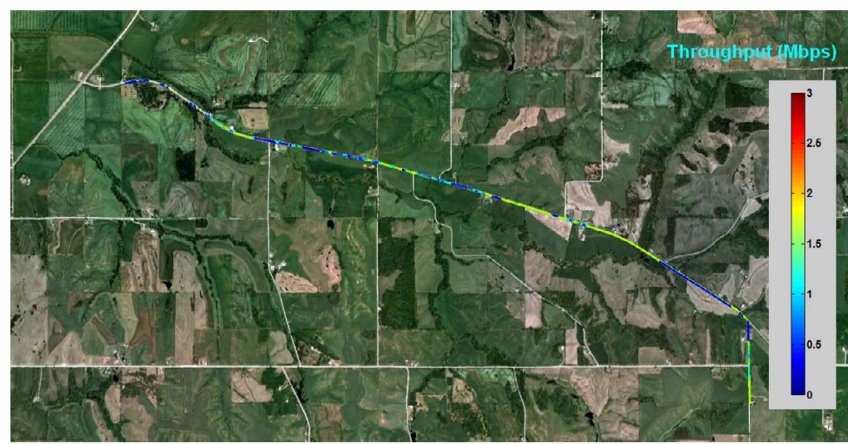

Figure 10. GPS information with uplink throughput results in Logan, IA.
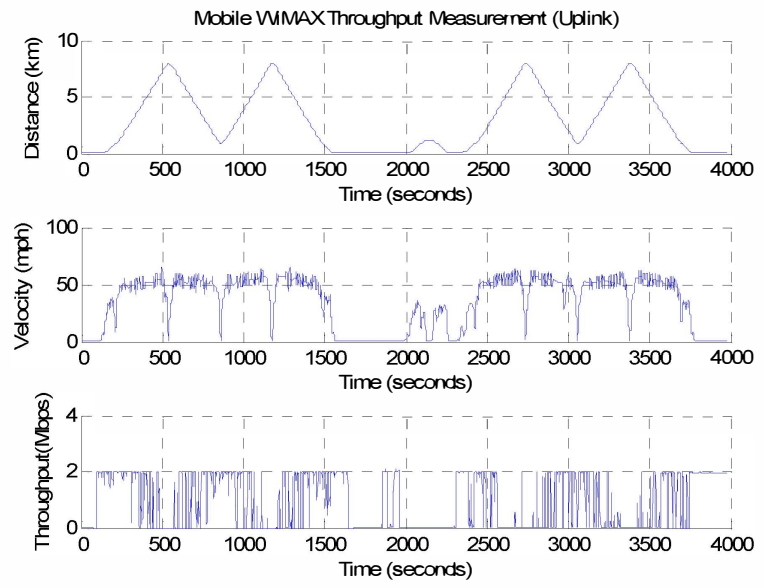

Figure 9. Uplink time domain measurement results in Logan, IA.

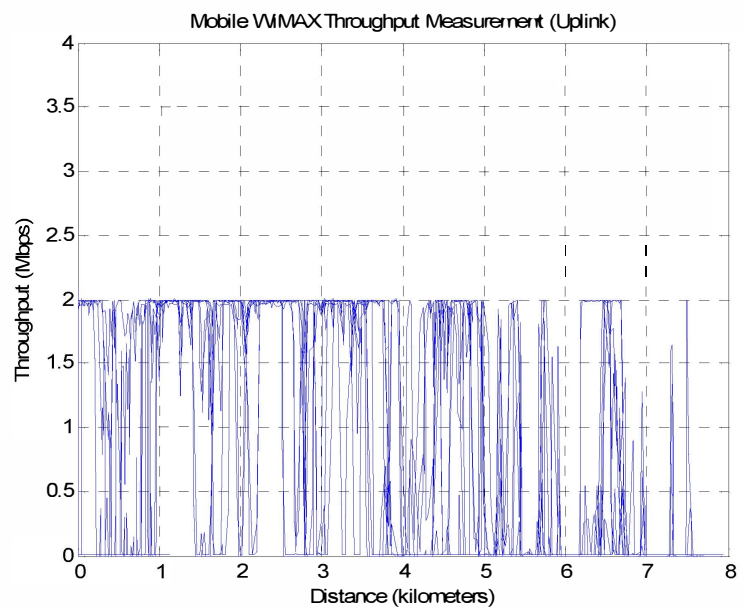

Figure 5. Mobile WiMAX uplink throughput and distance in Logan, IA.

information of this test with the color path showing the amount of measured throughput in Fig. 5. The throughput, velocities, and distances measured between the base station and the mobile client are shown in Fig. 6. At the beginning of this test, the car was close to the base station, and then moved away along the rural highway. After the client was out of communication range and lost the connection, we 
turned around (at the 600th second and the 1250th second in Fig. 6) and moved back towards the location of the base station. Most of the time, we tried to keep the velocity at 60 mph. Fig. 7 illustrates our downlink Mobile WiMAX throughput measurement at the Logan site with respect to distance in kilometers. It is obvious that the mobile downlink performance is quite stable at $2.8 \mathrm{Mbps}$ and the radio coverage can be up to 7.5 kilometers in this typical rural area. Some parts of the test road are blocked by trees and hills. At the point where the car lost its connection to the base station, the test road curves behind a hill effectively blocked signal reception. By the time we moved out from behind the RF shadow of the hill, we also ran into an intersection with another highway. If the test road is opened and is longer, we can expect the coverage to be larger.

In the second test in Logan, we measured the mobile uplink performance. This time the $5 \mathrm{Mbps}$ UDP packet traffic was transmitted back from the subscriber station to the base station instead. The subscriber station's transmit power was set to $25 \mathrm{dBm}$, which is the maximum value we can go. Similar to the downlink, we mapped GPS information of the uplink test with the color path showing the measured throughput in Fig. 8, while the throughput, velocities, and distances measured between the base station and the mobile client are demonstrated in Fig. 9. To make our measurements consistent, we also kept the velocity of the car at $60 \mathrm{mph}$. Fig. 10 shows our uplink Mobile WiMAX throughput measurement with respect to distance in kilometers. It is obvious that the mobile uplink throughput is less stable than the downlink throughput and is topped at $2 \mathrm{Mbps}$. The radio coverage can still be up to 7.5 kilometers in this road test.

Not only throughput, we also measured end-to-end latency in the mobile environment. We transmitted small packets with time stamp from the base station to the subscriber station. After the subscriber station received the packets, it immediately generated reply messages back to the base station. When the base station received these replies, it calculated the end-to-end delay as half of the roundtrip duration. Fig. 11 illustrates our latency measurement at different distances. The latency is more stable when the distance between the base station and the subscriber station is short. At the farther distances, the link qualities clearly affect the latency. We experienced some lost packets and retransmission mechanism also play an important part here. If the retransmission reaches limit, the packet shall be discarded.

\section{CONCLUSION}

In this paper, we investigated actual Mobile WiMAX performance from the certified Mobile WiMAX devices. We evaluated throughput performance and latency over a Mobile WiMAX test bed in Logan, Iowa, comprising one base station and one subscriber station. We employed a UDP traffic source and measured the throughput capacity of our WiMAX equipments in both downlink and uplink directions. The throughput, velocities, and distances were collected. We found that, using best effort scheduling, we can achieve a throughput of approximately $2.8 \mathrm{Mbps}$ downlink and $2 \mathrm{Mbps}$

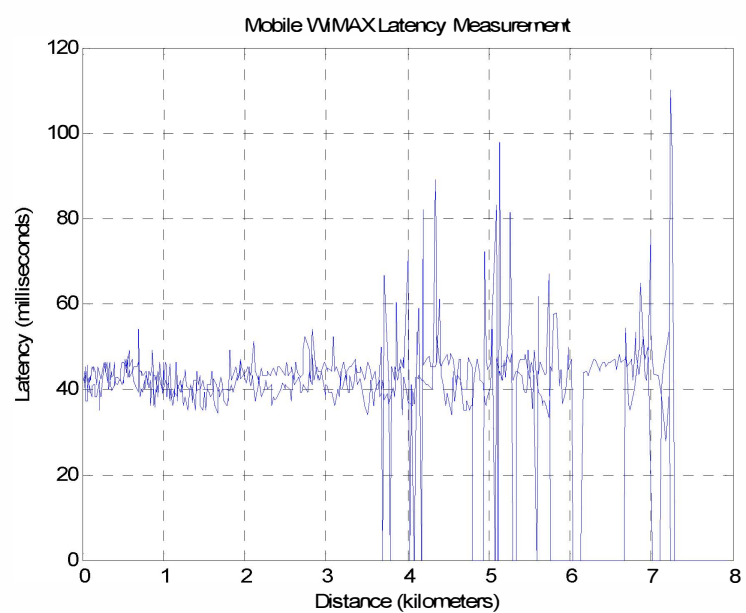

Figure 11. Mobile WiMAX throughput and distance in Logan, IA.

uplink with the QPSK 1/2 modulation scheme, a maximum distance of 7.5 kilometers, and a speed of $60 \mathrm{mph}$. These numbers make Mobile WiMAX a promising candidate for large-scale deployments in environments such as the railroad industry. We hope that our results provide a comprehensive measurement that will assist others in understanding Mobile WiMAX performance and guiding future network deployments.

\section{ACKNOWLEDGMENT}

This study was conducted at the University of NebraskaLincoln by the Advanced Telecommunications Engineering Laboratory (www.TEL.unl.edu) researchers, and funded by the FRA under the direction of Terry Tse. This study would not have been possible without the excellent support from Union Pacific Railroad, BNSF Railway, and the Association of American Railroads (AAR).

\section{REFERENCES}

[1] IEEE Standard 802.16. Part 16: Air interface for fixed broadband wireless access systems, 2004.

[2] IEEE Standard 802.16e. Part 16: Air interface for fixed and mobile broadband wireless access systems, 2006.

[3] Federal Railroad Administration. [Online] http://www.fra.dot.gov.

[4] M. Hempel, H. Sharif, T. Zhou, and P. Mahasukhon, "A wireless test bed for mobile 802.11 and beyond," in Proc. of IWCMC'06, pp. 1003-1008, July 2006.

[5] D. Kim, H. Cai, M. Na, and S. Choi, "Performance measurement over Mobile WiMAX/IEEE 802.16e network," in Proc. of WoWMoM'08, pp. 1-8, June 2008

[6] M. Tran, G. Zaggoulos, A. Nix, and A. Doufexi, "Mobile WiMAX: Performance analysis and comparison with experimental results," in Proc. of VTC'08-Fall, pp. 1-5, September 2008.

[7] A. Durantini, M. Petracca, and F. Ananasso, "Experimental evaluation of IEEE 802.16 WiMAX performances at $2.5 \mathrm{GHz}$ band," in Proc. of IWCMC'08, pp. 338-343, August 2008.

[8] R. G. Garroppo, S. Giordano, and D. Iacono, "Experimental and simulation study of a WiMAX system in the sea port scenario," in Proc. of ICC'09, pp. 1-5, June 2009.

[9] WiMAX Forum. [Online] http://www.wimaxforum.org. 